\title{
Pregnenolone, a cholesterol metabolite, induces glioma cell apoptosis via activating extrinsic and intrinsic apoptotic pathways
}

\author{
XIAO XIAO $^{1 *}$, LIJUN CHEN $^{1 *}$, YING OUYANG $^{2 *}$, WENBO ZHU $^{1}$, PENGXIN QIU $^{1}$, \\ XINWEN SU ${ }^{1}$, YUNLING DOU ${ }^{3}$, LIPENG TANG ${ }^{1}$, MIN YAN ${ }^{1}$, \\ HAIPENG ZHANG ${ }^{1}$, XIAOXIAO YANG ${ }^{1}$, DONG XU ${ }^{1}$ and GUANGMEI YAN ${ }^{1}$
}

${ }^{1}$ Department of Pharmacology, Zhongshan School of Medicine, Sun Yat-sen University, Guangzhou, Guangdong 510080;

${ }^{2}$ Department of Neonatology, Sun Yat-sen Memorial Hospital, Sun Yat-sen University, Guangzhou, Guangdong 510120;

${ }^{3}$ Department of Anesthesiology, The First Affiliated Hospital of Sun Yat-sen University, Guangzhou, Guangdong 510080, P.R. China

Received July 24, 2013; Accepted February 7, 2014

DOI: $10.3892 / 01.2014 .2147$

\begin{abstract}
Gliomas are one of the most common types of malignant tumors worldwide, however, an effective therapeutic strategy not yet been fully determined. The present study aimed to investigate the anti-glioma activity and underlying mechanisms of pregnenolone, which originates from cholesterol and is metabolized into important steroid hormones in the body. The results demonstrated that $100 \mu \mathrm{M}$ pregnenolone induced a significant loss of cell viability in various malignant glioma cell lines. In the U-87 MG, LN-18 and C6 cell lines, the loss of cell viability resulted from cell apoptosis, which was evidenced by apoptotic nuclear morphology changes and caspase 3 activation. Moreover, the increased activities of caspase 8 and 9 strongly indicated that pregnenolone activated the extrinsic and intrinsic pathways of apoptosis. Additionally, glioma cell apoptosis was prevented by the general caspase inhibitor, Z-VAD-FMK. In the C6 cells, upregulation of Fas and Fas ligand triggered the activation of the extrinsic pathway, whereas knockdown of Fas significantly abrogated the cell apoptosis that was induced by pregnenolone. Furthermore, downregulation of the anti-apoptotic protein, B-cell lymphoma 2 and upregulation of pro-apoptotic proteins, such as Bax and Bak, activated the intrinsic pathway. In conclusion, pregnenolone induced glioma cell apoptosis in a caspase-dependent manner, which was mediated by activation of the extrinsic and intrinsic apoptotic pathways.
\end{abstract}

Correspondence to: Professor Guangmei Yan or Dr Dong Xu, Department of Pharmacology, Zhongshan School of Medicine, Sun Yat-sen University, 74 Zhongshan Road II, Guangzhou, Guangdong 510080, P.R. China

E-mail:ygm@mail.sysu.edu.cn

E-mail: xudong@mail.sysu.edu.cn

*Contributed equally

Key words: pregnenolone, glioma, apoptosis

\section{Introduction}

Gliomas originate from glial cells and are the most common type of primary brain tumors accounting for $80 \%$ of all malignant primary brain tumors (1). According to the pathological and clinical criteria established by the World Health Organization, gliomas are classified as grades I-IV $(2,3)$. Grade IV tumors, such as glioblastomas (GBMs), are the most devastating and aggressive form comprising $>50 \%$ of gliomas, and have a poor prognosis (4). The current treatment standard of GBMs is surgical resection to a feasible extent, followed by radiotherapy and chemotherapy. Among the currently available chemotherapy agents, temozolomide is the most popular; doctors and patients favor it as it is administrated orally and efficiently crosses the blood-brain barrier (BBB) (5). However, $67.2-76 \%$ of patients are resistant to this agent and therefore do not benefit from it (6). Regardless of systemic therapeutic strategies, including surgery, temozolomide and radiotherapy, patient median survival is only 14.6 months and the five-year survival rate is $\sim 9.8 \%(7,8)$. The poor prognosis of glioma fuels the requirement for identifying therapeutic agents with the merits of temozolomide, such as high lipophilicity and strong anti-glioma activity.

Steroid hormones are generally divided into the following five groups: Estrogens, androgens, progestogens, glucocorticoids and mineralocorticoids $(9,10)$. The natural steroid hormones are predominantly synthesized from cholesterol in the gonads and adrenal glands, and readily diffuse through the cell membrane due to their lipophilic properties $(11,12)$. Accumulating evidence has indicated that certain steroid hormones possess antitumor activities, such as the $17 \beta$-estradiol metabolite, 2-methoxyestradiol (2ME), which exerts the strongest activity. 2ME inhibits proliferation and induces apoptosis of various types of cancer, including gliomas, and breast and gastric cancer, independently of estrogen receptors $\alpha$ and $\beta$ (13-15). However, findings from a clinical trial identified low oral bioavailability of $2 \mathrm{ME}$, which prevents the transfer of this promising agent from bench to bed side (16). The example of $2 \mathrm{ME}$ implies the application potential of steroid hormones and provides the basis for the subsequent 
investigation of other endogenous steroid hormones, such as pregnenolone (Fig. 1).

The present study aimed to investigate the pharmacological effects of the endogenous steroid, pregnenolone, on GBM cells, and the mechanisms underlying its pro-apoptotic activity via the extrinsic and intrinsic apoptotic pathways. Pregnenolone may be a leading compound in the treatment of gliomas and may be modified and developed for clinical application.

\section{Materials and methods}

Antibodies and reagents. Antibodies against tubulin, B-cell lymphoma 2 (Bcl-2) and Fas ligand (L) were obtained from Cell Signaling Technology, Inc. (Beverly, MA, USA). Bak and Bax antibodies were purchased from Santa Cruz Biotechnology, Inc. (Santa Cruz, CA, USA) and cluster of differentiation (CD)95/Fas antibody was purchased from Epitomics, Inc. (Burlingame, CA, USA). Pregnenolone, Hoechst 33342 and methyl-thiazolyl-tetrazolium (MTT) were purchased from Sigma-Aldrich (St. Louis, MO, USA). The general caspase inhibitor, Z-VAD-FMK, was obtained from EMD Millipore Corp. (Billerica, MA, USA). The Caspase-Glo ${ }^{\circledR}$ 8, 9 and 3/7 Activity assay kits were purchased from Promega Corp. (Madison, WI, USA).

Cell culture and drug treatment. The C6 rat glioma and U-87 MG and LN-18 human glioma cell lines were obtained from the American Type Culture Collection (Manassas, VA, USA). The cells were cultured in Dulbecco's modified Eagle's medium (DMEM; Gibco, Grand Island, NY, USA) supplemented with fetal bovine serum (FBS; Invitrogen Life Technologies, Carlsbad, CA, USA), 100X MEM Non-Essential Amino Acid, GlutaMax ${ }^{\mathrm{TM}}$ (Gibco), penicillin (Gibco) and streptomycin (Gibco) in a humidified atmosphere of $5 \% \mathrm{CO}_{2}$ at $37^{\circ} \mathrm{C}$. Pregnenolone was dissolved in ethanol to obtain a $10-\mathrm{mM}$ stock solution and stored at $-20^{\circ} \mathrm{C}$. For the drug treatment, pregnenolone was diluted in DMEM and added at different concentrations. Ethanol at corresponding concentrations served as the vehicle control.

Cell viability and cytotoxicity assays. Cell viability was determined via MTT assay. The cells that were growing in logarithmic phase were seeded in 96-well plates and treated with pregnenolone. MTT $(10 \mu \mathrm{l} ; 0.5 \mathrm{mg} / \mathrm{ml})$ was added to each well, which was subsequently incubated at $37^{\circ} \mathrm{C}$ for $4 \mathrm{~h}$ to allow the yellow dye to transform into blue crystals. The medium was removed and $100 \mu$ l dimethyl sulfoxide (DMSO; Sigma-Aldrich) was added to each well to dissolve the dark blue crystals. The optical density was measured with a microplate reader (iMark ${ }^{\mathrm{TM}}$, Bio-Rad, Hercules, CA, USA) at $490 \mathrm{~nm}$. Five replicates were prepared for each condition.

Lactate dehydrogenase (LDH) release was quantified with a CytoTox $96{ }^{\circledR}$ Non-Radioactive Cytotoxicity assay kit (Promega Corp.) according to the manufacturer's instructions. Plates were incubated with an LDH substrate at room temperature for $30 \mathrm{~min}$ in the dark and absorbance was measured at $490 \mathrm{~nm}$ with a microplate reader (iMark ${ }^{\mathrm{TM}}$, Bio-Rad).

Caspase 3/7, 8 and 9 activities assay. The glioma cells were seeded into 96-well plates at 3,000-5,000 cells/well and incubated for $24 \mathrm{~h}$. The cells were treated with pregnenolone at different concentrations. After $24 \mathrm{~h}$ of treatment, the enzymatic activity of caspase 8,9 and 3/7 was determined using the Caspase-Glo ${ }^{\circledR} 8$ and 9 Activity assay kit and the Caspase-Glo ${ }^{\circledR}$ 3/7 Activity assay kit, respectively, according to the manufacturer's instructions. Simultaneously, the cells were plated in 96-well plates and treated with pregnenolone for $24 \mathrm{~h}$. The relative cell number was measured by MTT assay and evaluated as follows: Caspase activities = total enzymatic activity values of caspase/cell number.

Hoechst 33342 staining and terminal deoxynucleotidyl transferase dUTP nick end labeling (TUNEL) assay. The glioma cells were stained with Hoechst $33342(5 \mu \mathrm{g} / \mathrm{ml})$ at $37^{\circ} \mathrm{C}$ for $20 \mathrm{~min}$ in the dark and photographed under a fluorescent microscope (IX71, Olympus Corp., USA) with a 340-nm excitation filter. Apoptotic cells were characterized as demonstrating condensed nuclei and cell shrinkage. The proportion of apoptotic cells in total cells was quantified in three randomly selected microscopic fields.

For the TUNEL assay (Roche Diagnostics $\mathrm{GmbH}$, Mannheim, Germany), the glioma cells were fixed in $4 \%$ paraformaldehyde solution at room temperature for $1 \mathrm{~h}$. The cell samples were detected via an In Situ Cell Death Detection kit, TMR red (Roche Diagnostics $\mathrm{GmbH}$ ) according to the manufacturers' instructions and analyzed under a fluorescent microscope (Olympus Corp.) with a 540-nm excitation filter. A red fluorescence signal was observed in the apoptotic cells.

Immunoblotting. Immunoblotting was performed as previously described (17). Briefly, following cell lysis and measurement of protein concentrations, the cells were dissolved in sodium dodecyl sulfate (SDS) sample buffer (Beyotime Institute of Biotechnology, Shanghai, China). Equal amounts of protein were analyzed by SDS-PAGE on $12 \%$ polyacrylamide gels (Bio-Rad) and the proteins were electroblotted onto polyvinylidene fluoride membranes (Roche Diagnostics GmbH). The membranes were incubated in 5\% non-fat dry milk in Tris-buffered saline ( $\mathrm{NaCl} / \mathrm{Tris}$; Boster Biological Engineering Co., Ltd., Wuhan, China) containing $0.1 \%$ Tween-20 (Sigma-Aldrich) for $1 \mathrm{~h}$ at room temperature overnight at $4^{\circ} \mathrm{C}$, and subsequently incubated with primary antibodies. Following incubation with a horseradish peroxidase-labeled secondary antibody, protein exposure was achieved with a molecular imager (ChemiDOC ${ }^{\text {TM }} \mathrm{XRS}+$, Bio-Rad).

Small interfering (si)RNA-mediated knockdown of Fas expression. Fas-siRNA (si-Fas) and negative control (NC) oligonucleotides were purchased from Guangzhou RiboBio Co., Ltd. (Guangzhou, China). The C6 cells of 30-50\% confluence were transfected by Lipofectamine ${ }^{\circledR}$ RNAiMAX (Invitrogen Life Technologies) according to the manufacturer's instructions and siRNA inhibitory efficacy was examined by immunoblotting.

Statistical analysis. Data are expressed as means \pm standard deviation of the three separate experiments. One-way analysis of variance and Student's t-test were used to determine the differences between the the two groups. $\mathrm{P}<0.05$ was considered to indicate a statistically significant difference. 

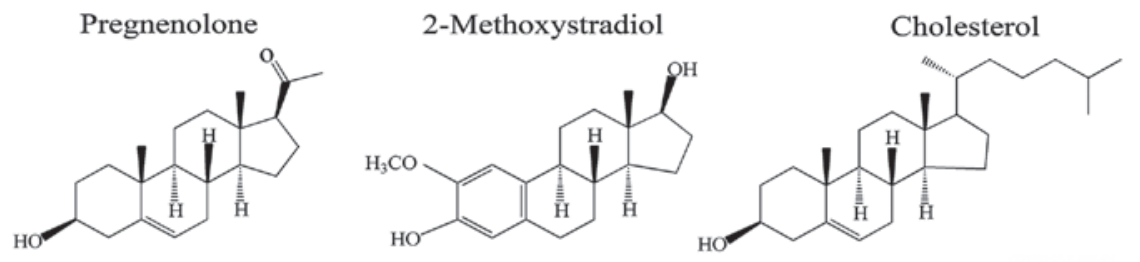

Figure 1. Chemical structure of pregnenolone and 2-methoxyestradiol, the metabolites of cholesterol.
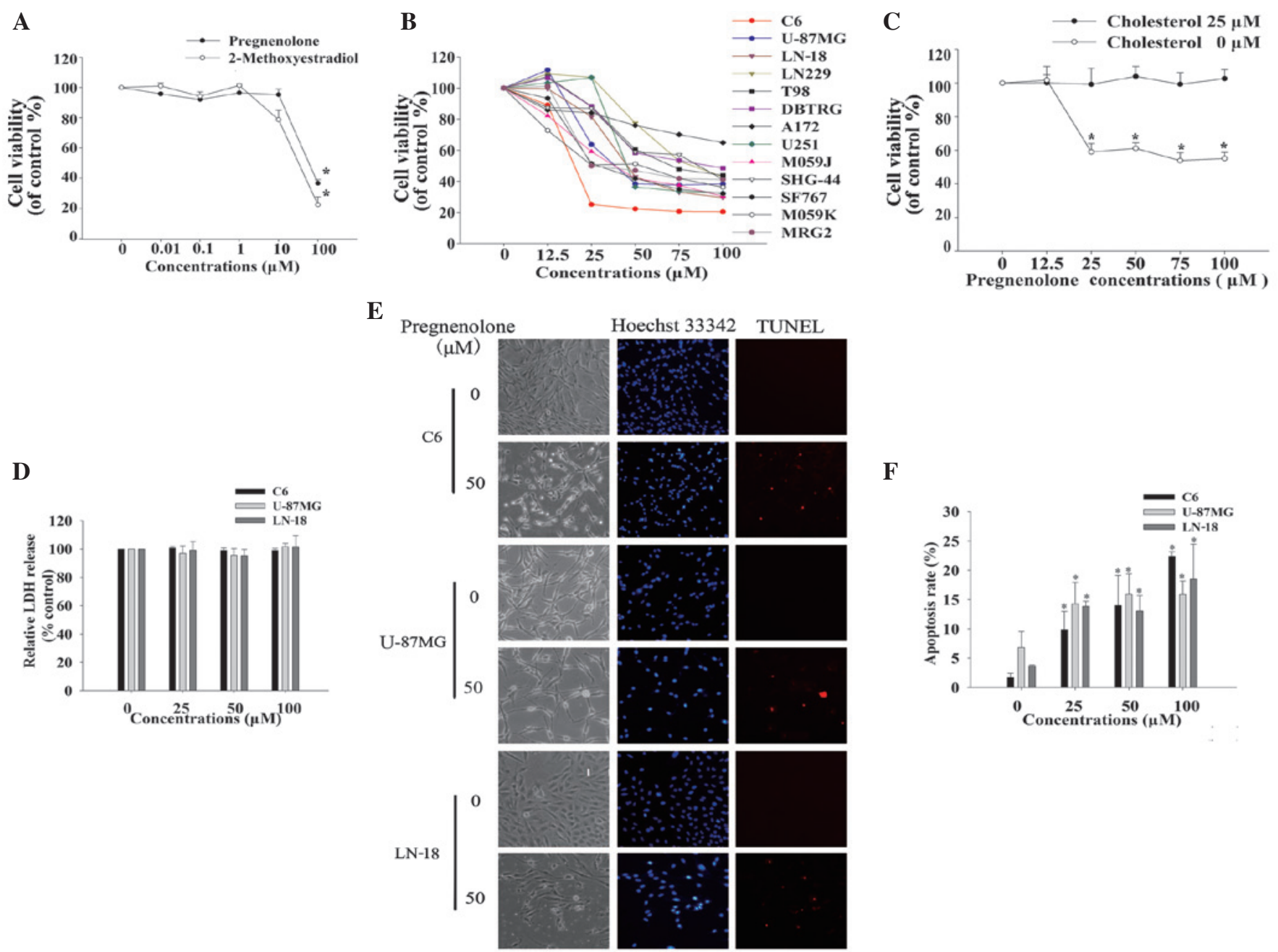

Figure 2. Pregnenolone decreased cell viability in glioma cells by inducing apoptosis. (A) Glioma cells were treated with different doses of pregnenolone for $48 \mathrm{~h}$. Cell viability was determined by the MTT assay. Similar to $2 \mathrm{ME}$, pregnenolone decreased cell viability at $100 \mu \mathrm{M}$ in the U-87 MG human glioma cell line $\left(\mathrm{n}=3\right.$; ${ }^{\prime} \mathrm{P}<0.001$, Bonferroni t-test). (B) Pregnenolone decreased cell viability at $48 \mathrm{~h}$ in a series of glioma cell lines. (C) Exogenous cholesterol ( $\left.25 \mu \mathrm{M}\right)$ prevented the pregnenolone-induced cell loss in U-87 MG cells at $48 \mathrm{~h}\left({ }^{*} \mathrm{P}<0.05\right)$. (D) The C6, LN-18 and U-87 MG glioma cells were treated with different concentrations of pregnenolone for $48 \mathrm{~h}(\mathrm{n}=3)$. No significant LDH release changes were observed in the cytotoxicity assay. (E) Pregnenolone induced glioma cell apoptosis after $48 \mathrm{~h}$ showing characteristics of nuclear condensation and DNA breakage. (F) Bar chart of the apoptosis rate (\%) in glioma cells at $48 \mathrm{~h}\left({ }^{*} \mathrm{P}<0.05\right)$. $2 \mathrm{ME}$, 2-methoxyestradiol; LDH, lactate dehydrogenase; TUNEL, terminal deoxynucleotidyl transferase dUTP nick end labeling. "P<0.001 vs $0 \mu \mathrm{M}$ pregnenolone.

\section{Results}

Pregnenolone decreases cell viability of malignant glioma cells. First, the effect of pregnenolone on the growth of U-87 MG human GBM cells was investigated. The cell numbers were greatly reduced following treatment with $100 \mu \mathrm{M}$ pregnenolone for $48 \mathrm{~h}$ (Fig. 2A) and the growth inhibitory efficacy was comparable with 2-ME. To further investigate the dose-dependent effect of pregnenolone on the anti-glioma spectrum, a series of glioma cell lines, including
C6, LN-18 and T98, were treated with pregnenolone at different doses $(0,12.5,25,50,75$ and $100 \mu \mathrm{M})$ for $48 \mathrm{~h}$. Notably, pregnenolone dose-dependently decreased the cell viability in all the glioma cell lines at varying degrees (Fig. 2B). The dose-dependent decline of cell viability in the majority of the cell lines was more sensitive between 12.5 and $50 \mu \mathrm{M}$ pregnenolone compared with between 50 and $100 \mu \mathrm{M}$ pregnenolone. Therefore, the glioma cells were treated with doses between 12.5 and $50 \mu \mathrm{M}$ pregnenolone to further investigate the detailed mechanisms of action. Exogenous cholesterol 
A
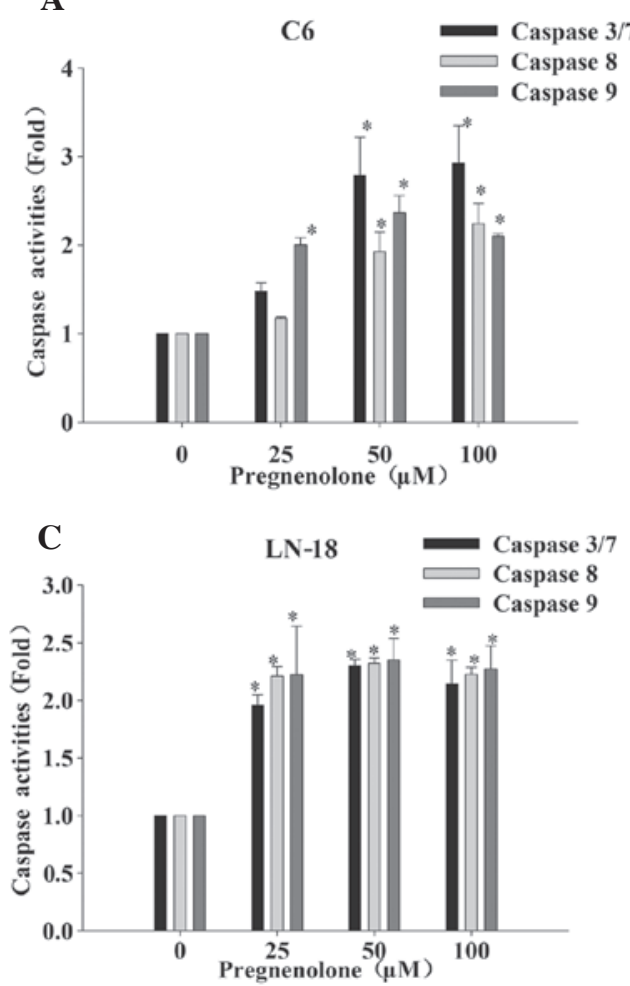

B
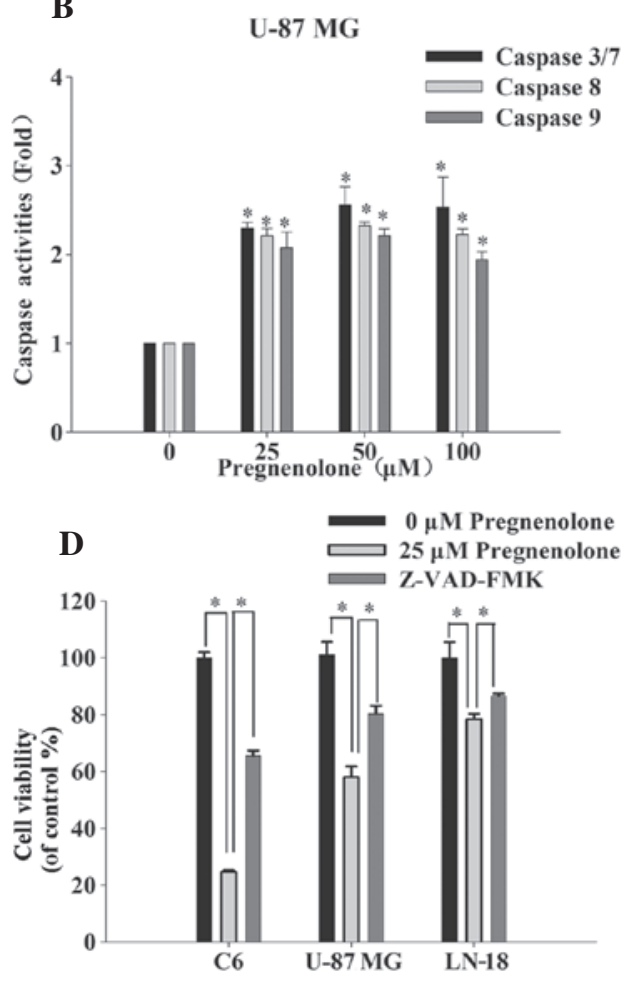

Figure 3. Pregnenolone induced apoptosis by the activation of caspases. The C6, LN18 and U-87MG glioma cells were incubated with different concentrations $(0,25,50$ and $100 \mu \mathrm{M})$ of pregnenolone for $48 \mathrm{~h}(\mathrm{n}=3)$. The activation of caspase 3/7, 8 and 9 were observed in (A) C6, (B) U-87 MG and (C) LN-18 ("P<0.05). (D) General caspase inhibitor, Z-VAD-FMK, at $50 \mu \mathrm{M}$ partly prevented glioma cell apoptosis induced by pregnenolone. ${ }^{*} \mathrm{P}<0.05 \mathrm{vs}$. $0 \mu \mathrm{M}$ pregnenolone group.

may completely prevent the pregnenolone-induced cell loss in U-87MG cells (Fig. 2C).

Pregnenolone induces glioma cell apoptosis. To investigate the mechanisms underlying the loss of cell viability induced by pregnenolone, further experiments in the C6, U-87 MG and LN-18 glioma cell lines were conducted. The glioma cells were incubated with pregnenolone at different concentrations for $48 \mathrm{~h}$, and examined via cytotoxicity assay, Hoechst 33342 staining and TUNEL assay. No significant changes in LDH release were observed in the pregnenolone-treated glioma cells, indicating that pregnenolone did not result in cell necrosis (Fig. 2D). Compared with the control group, the condensed chromatin in the nucleus of the $50 \mu \mathrm{M}$ pregnenolone-treated group appeared smaller and brighter under the fluorescent microscope (Fig. 2E). The apoptosis rate in the pregnenolone-treated groups increased in a dose-dependent manner (Fig. 2F). Following the TUNEL assay, there were more positive signals observed in the cells that were treated with pregnenolone (Fig. 2E). These findings support the conclusion that pregnenolone induced glioma cell apoptosis in a dose-dependent manner.

Pregnenolone leads to caspase-dependent apoptosis via extrinsic and intrinsic apoptotic pathways. Previous studies have reported that anticancer therapies eventually result in the activation of caspases, a family of cysteine proteases that act as common death effector molecules in the apoptotic pathway (18-20). To determine whether the apoptotic pathway is induced by pregnenolone, the activity of caspase 8 and 9, and 3/7 in the death receptor (extrinsic) and mitochondria-dependent (intrinsic) pathways, respectively, was investigated. Caspase 9 is an important intracellular amplifier of caspase signaling that is downstream of mitochondria and caspase 8 is an apical caspase in death receptor signaling, which has been well-established (21). After $24 \mathrm{~h}$ of exposure to pregnenolone, the activity of caspase 8 and 9 markedly increased compared with the control group. The activity of the downstream effector caspase 3/7 increased 2-3-fold following a 48 -h exposure to pregnenolone (Fig. 3A-C). To determine whether apoptosis was due to the activation of caspases, the general caspase inhibitor, Z-VAD-FMK, was applied to the pregnenolone-treated glioma cells and cell viability was measured via an MTT assay. As expected, $50 \mu \mathrm{M}$ Z-VAD-FMK prevented the loss of cell viability that was caused by pregnenolone (Fig. 3D).

Pregnenolone induces apoptosis by decreasing Bcl-2 and increasing Fas/FasL activity. The present study demonstrated that pregnenolone triggers the intrinsic and extrinsic apoptotic pathways by activating caspase 8 and 9. To investigate the mechanisms underlying pregnenolone-induced cell apoptosis, the upstream targets in the mitochondria- and death receptor-mediated pathways were analyzed. First, the protein levels of the Bcl-2 family members, which are responsible for the stability of the mitochondrial membrane, were examined. The data demonstrated that the anti-apoptotic protein, Bcl-2, was markedly downregulated, while the pro-apoptotic factors, Bax and Bak, were upregulated in the pregnenolone-treated C6 cells (Fig. 4A). In addition, 
A

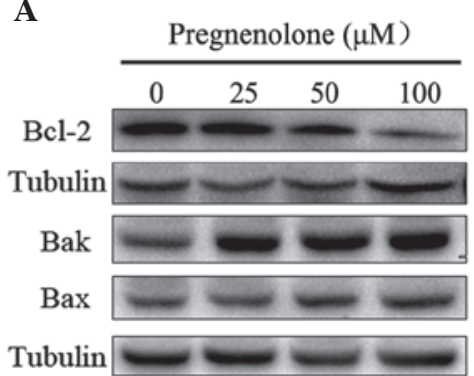

B

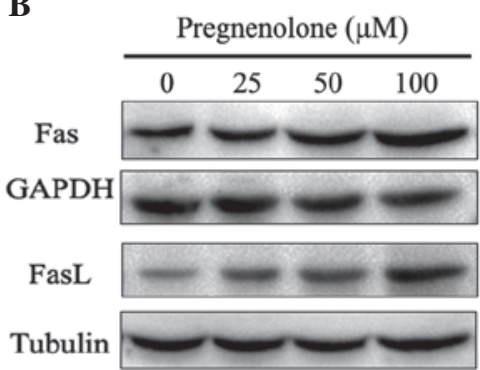

C

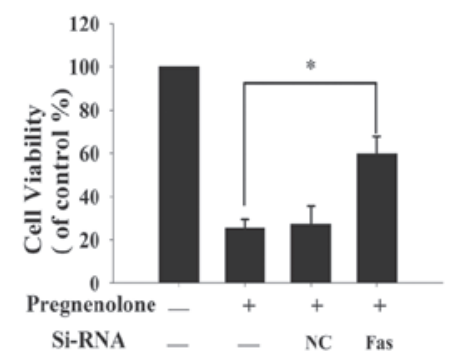

Figure 4. Pregnenolone-induced apoptosis was mediated by regulating Fas, FasL and Bcl-2 family members. Western blot analysis was performed in C6 cells after $48 \mathrm{~h}$ of incubation with pregnenolone at different concentrations ( $\mathrm{n}=3$ ). (A) Pregnenolone decreased the protein expression of Bcl-2 and increased the levels of Bak and Bax. (B) Upregulation of Fas and FasL was observed in the pregnenolone-treated groups. (C) Knockdown of Fas partly rescued the cell viability-loss by pregnenolone. ${ }^{*} \mathrm{P}<0.05$ vs. pregnenolone-treated group without siRNA. Bcl-2, B-cell lymphoma 2; FasL, fas ligand; siRNA, small interfering RNA.

pregnenolone treatment resulted in a marked increment of the activity of Fas and FasL, an important death receptor and ligand, respectively that are involved in the extrinsic pathway (Fig. 4B). To confirm whether pregnenolone-induced apoptosis was mediated by Fas and FasL, Fas was silenced in the C6 cells, using siRNA, and the cell viability was measured (Fig. 4D). Compared with the cell viability loss that was observed in the control group, Fas knockdown significantly decreased the cell apoptosis, which was induced by pregnenolone. These findings indicated that pregnenolone may trigger the intrinsic and extrinsic apoptotic pathways by regulating the Bcl-2 family, Fas and FasL.

\section{Discussion}

Pregnenolone, a neurosteroid, is synthesized in the nervous system $(22,23)$, arises from cholesterol and is metabolized into important steroid hormones (24). Due to its chemical structure, pregnenolone is highly lipid-soluble and rapidly crosses the BBB (25). The concentration of pregnenolone in overall brain tissue is 74-fold higher compared with that in plasma (26). Notably, the findings of the present study indicated that this endogenous steroid hormone is able to induce glioma cell apoptosis. The antitumor efficacy of pregnenolone is as strong as 2-ME, however, whether it may be developed into an anti-glioma agent requires further investigation, as does its bioavailability.

In addition to pregnenolone and 2-ME, previous studies have reported that a number of other steroid hormones originating from cholesterol exhibit antineoplastic properties, such as dehydroepiandrosterone (DHEA) (27) and cholesteryl sulfate (28), the underlying mechanisms of which are distinct. Furthermore, DHEA induced apoptotic and necrotic death in cervical cancer cells (29). In the present study, pregnenolone induced glioma cell apoptosis, but not necrotic death. Additionally, 2-ME upregulated death receptor 5 and induced apoptosis through activation of the extrinsic apoptotic pathway (13). However, the present study demonstrated that pregnenolone upregulated Fas and FasL, and induced apoptosis through the activation of the extrinsic pathway. Moreover, the intrinsic apoptotic pathway was activated by pregnenolone, which was characterized by downregulation of anti-apoptotic Bcl-2 expression, upregulation of pro-apoptotic Bax and Bak expression, and activation of caspase 9.
Notably, cholesterol blocked the loss of cell viability that was induced by pregnenolone (Fig. 2C), demonstrating the critical role of cholesterol in apoptosis. As a neutral lipid, cholesterol is indispensable in the regulation of cell membrane properties in mammalian cells, contributes to the unique biophysical properties of the lipid raft microdomain and is mechanistically important for signal transduction by raft proteins (30). Lipid rafts, incorporating distinct classes of proteins, are comprised of cholesterol and sphingolipids in the exoplasmic leaflets of the bilayer $(31,32)$. Disruption of lipid rafts by dispersion or extraction of membrane cholesterol results in inhibition of raft-dependent signaling events and eventually affects cell survival. It was reported that simvastatin, a cholesterol synthesis inhibitor, lowered raft cholesterol content and induced apoptosis in prostate cancer cells; however, replenishing cell membranes with cholesterol reversed these inhibitory and pro-apoptotic effects (33). In addition, previous studies have shown that ginsenoside Rh2, containing a cholesterol backbone with hydroxyl groups, induced ligand-independent Fas activation and activated caspase 8 via lipid raft disruption $(34,35)$. Similar to Rh2, other cholesterol derivatives, such as desmosterol treatment, resulted in the disappearance of caveolae and blocked insulin receptor activation via lipid raft disruption (36). As a cholesterol metabolite, pregnenolone has a similar chemical structure to cholesterol, and may interrupt normal lipid rafts and trigger apoptosis.

The Fas/FasL and mitogen-activated protein kinase (MAPK) signaling pathways are two key cell death and survival signaling pathways, which are influenced by the alteration of lipid rafts (37). In the present study, Fas/FasL signaling was dramatically potentiated (Fig. 4), while EGFR/Ras/MAPK signaling remained stable (data not shown). Moreover, an elevation in Fas/FasL signaling was confirmed as a critical cause for pregnenolone-induced apoptosis by si-Fas. The mechanisms by which pregnenolone upregulates Fas and FasL, and whether pregnenolone disrupts lipid rafts require further investigation.

In conclusion, the present study demonstrated that pregnenolone, an endogenous steroid hormone, exerts a strong anti-glioma effect by inducing apoptosis. Pregnenolone treatment resulted in caspase-dependent apoptosis via the extrinsic and intrinsic apoptotic pathways in glioma cells. Based on this anti-glioma activity and high BBB permeability, 
pregnenolone may be a promising compound for, and aid in the development of, novel agents for anti-glioma therapy.

\section{Acknowledgements}

The present study was supported by the Natural Science Foundation of China (grant nos. 81202554 and 81202555) and the Guangdong Natural Science Foundation (grant nos. S10451008901004893 and S2012010009237).

\section{References}

1. Dolecek TA, Propp JM, Stroup NE and Kruchko C: CBTRUS statistical report: primary brain and central nervous system tumors diagnosed in the United States in 2005-2009. Neuro Oncol 14 Suppl 5: v1-v49, 2012.

2. Louis DN, Ohgaki H, Wiestler OD, et al: The 2007 WHO classification of tumours of the central nervous system. Acta Neuropathol 114: 97-109, 2007.

3. Yan H, Parsons DW, Jin G, et al: IDH1 and IDH2 mutations in gliomas. N Engl J Med 360: 765-773, 2009.

4. Rousseau A, Mokhtari K and Duyckaerts C: The 2007 WHO classification of tumors of the central nervous system - what has changed? Curr Opin Neurol 21: 720-727, 2008.

5. Mutter N and Stupp R: Temozolomide: a milestone in neuro-oncology and beyond? Expert Rev Anticancer Ther 6 : 1187-1204, 2006.

6. Chamberlain MC: Temozolomide: therapeutic limitations in the treatment of adult high-grade gliomas. Expert Rev Neurother 10 1537-1544, 2010.

7. Stupp R, Mason WP, van den Bent MJ, et al: Radiotherapy plus concomitant and adjuvant temozolomide for glioblastoma. N Engl J Med 352: 987-996, 2005.

8. Wirth T, Samaranayake H, Pikkarainen J, Määttä AM and Ylä-Herttuala S: Clinical trials for glioblastoma multiforme using adenoviral vectors. Curr Opin Mol Ther 11: 485-492, 2009.

9. Gorski J and Gannon F: Current models of steroid hormone action: a critique. Annu Rev Physiol 38: 425-450, 1976.

10. Stocco DM: StAR protein and the regulation of steroid hormone biosynthesis. Annu Rev Physiol 63: 193-213, 2001.

11. Falkenstein E, Tillmann HC, Christ M, Feuring $M$ and Wehling M: Multiple actions of steroid hormones - A focus on rapid, nongenomic effects. Pharmacol Rev 52: 513-555, 2000.

12. Rupprecht R: The neuropsychopharmacological potential of neuroactive steroids. J Psychiatr Res 31: 297-314, 1997.

13. LaVallee TM, Zhan XH, Johnson MS, et al: 2-methoxyestradiol up-regulates death receptor 5 and induces apoptosis through activation of the extrinsic pathway. Cancer Res 63: 468-475, 2003.

14. LaVallee TM, Zhang XGH, Herbstritt CJ, Kough EC, Green SJ and Pribluda VS: 2-methoxyestradiol inhibits proliferation and induces apoptosis independently of estrogen receptors alpha and beta. Cancer Res 62: 3691-3697, 2002

15. LinHL,Liu TY,Wu CW and ChiCW:2-Methoxyestradiol-induced caspase- 3 activation and apoptosis occurs through $\mathrm{G}(2) / \mathrm{M}$ arrest dependent and independent pathways in gastric carcinoma cells. Cancer 92: 500-509, 2001.

16. Kirches E and Warich-Kirches M: 2-methoxyestradiol as a potential cytostatic drug in gliomas? Anticancer Agents Med Chem 9: 55-65, 2009.

17. Tang YC, Williams BR, Siegel JJ and Amon A: Identification of aneuploidy-selective antiproliferation compounds. Cell 144: 499-512, 2011

18. Fulda S and Debatin KM: Extrinsic versus intrinsic apoptosis pathways in anticancer chemotherapy. Oncogene 25: 4798-4811, 2006.
19. Chandrasekar B, Vemula K, Surabhi RM, et al: Activation of intrinsic and extrinsic proapoptotic signaling pathways in interleukin-18-mediated human cardiac endothelial cell death. J Biol Chem 279: 20221-20233, 2004.

20. Johnstone RW, Frew AJ and Smyth MJ: The TRAIL apoptotic pathway in cancer onset, progression and therapy. Nat Rev Cancer 8: 782-798, 2008.

21. Degterev A, Boyce M and Yuan J: A decade of caspases. Oncogene 22: 8543-8567, 2003.

22. Dubrovsky BO: Steroids, neuroactive steroids and neurosteroids in psychopathology. Prog Neuropsychopharmacol Biol Psychiatry 29: 169-192, 2005.

23. Mellon SH and Griffin LD: Neurosteroids: biochemistry and clinical significance. Trends Endocrinol Metab 13: 35-43, 2002.

24. Marx CE, Bradford DW, Hamer RM, et al: Pregnenolone as a novel therapeutic candidate in schizophrenia: emerging preclinical and clinical evidence. Neuroscience 191: 78-90, 2011.

25. Tsutsui K, Matsunaga M and Ukena K: Biosynthesis and biological actions of neurosteroids in the avian brain. Avian Poultry Biol Rev 14: 63-78, 2003.

26. Lacroix C, Fiet J, Benais JP, et al: Simultaneous radioimmunoassay of progesterone, androst-4-enedione, pregnenolone, dehydroepiandrosterone and 17-hydroxyprogesterone in specific regions of human brain. J Steroid Biochem 28: 317-325, 1987.

27. Liu S, Ishikawa H, Li FJ, et al: Dehydroepiandrosterone can inhibit the proliferation of myeloma cells and the interleukin-6 production of bone marrow mononuclear cells from patients with myeloma. Cancer Res 65: 2269-2276, 2005.

28. Ishimaru C, Yonezawa Y, Kuriyama I, Nishida M, Yoshida H and Mizushina Y: Inhibitory effects of cholesterol derivatives on DNA polymerase and topoisomerase activities, and human cancer cell growth. Lipids 43: 373-382, 2008.

29. Girón RA, Montaño LF, Escobar ML, López-Marure R: Dehydroepiandrosterone inhibits the proliferation and induces the death of HPV-positive and HPV-negative cervical cancer cells through an androgen- and estrogen-receptor independent mechanism. FEBS J 276: 5598-5609, 2009.

30. Simons K and Ikonen E: How cells handle cholesterol. Science 290: 1721-1726, 2000.

31. Lingwood D and Simons K: Lipid rafts as a membrane-organizing principle. Science 327: 46-50, 2010.

32. Anderson RG and Jacobson K: A role for lipid shells in targeting proteins to caveolae, rafts, and other lipid domains. Science 296: 1821-1825, 2002.

33. Zhuang L, Kim J, Adam RM, Solomon KR and Freeman MR: Cholesterol targeting alters lipid raft composition and cell survival in prostate cancer cells and xenografts. J Clin Invest 115: 959-968, 2005.

34. Shieh PC, Tsao CW, Li JS, et al: Role of pituitary adenylate cyclase-activating polypeptide (PACAP) in the action of ginsenoside $\mathrm{Rh} 2$ against beta-amyloid-induced inhibition of rat brain astrocytes. Neurosci Lett 434: 1-5, 2008.

35. Yi JS, Choo HJ, Cho BR, et al: Ginsenoside Rh2 induces ligand-independent Fas activation via lipid raft disruption. Biochem Biophys Res Commun 385: 154-159, 2009.

36. Jansen M, Pietiaïnen VM, Pölönen H, et al: Cholesterol substitution increases the structural heterogeneity of caveolae. J Biol Chem 283: 14610-14618, 2008.

37. Patra SK: Dissecting lipid raft facilitated cell signaling pathways in cancer. Biochim Biophys Acta 1785: 182-206, 2008. 